\title{
Erratum: Rashba scattering in the low-energy limit [Phys. Rev. B 93, 245309 (2016)]
}

Joel Hutchinson and Joseph Maciejko

(Received 19 December 2016; published 11 January 2017)

DOI: 10.1103/PhysRevB.95.039902

In this Erratum, we correct a typographical error that occurred in writing the low-energy limit of the $S$ matrix for scattering from a hard disk [Eq. (27)]. In the paragraph directly above this equation, the off-diagonal coefficient is correctly stated as $b_{l}=-i^{l} / 2 \sqrt{2}$. This means that the correct expression for the low-energy $S$ matrix is, in fact,

$$
S^{l}=\left(\begin{array}{cc}
0 & -1 \\
-1 & 0
\end{array}\right) \text {. }
$$

This mistake was reflected in the conclusion [Sec. V, paragraph 1] where its was stated "the $S$ matrix approaches a purely off-diagonal form with both off-diagonal elements equal to one," which of course should read "the $S$ matrix approaches a purely off-diagonal form with both off-diagonal elements equal to negative one."

The same sign error appeared one last time in the second paragraph of Sec. III B: "Using the observation that in this limit, $c_{l}=\tilde{b}_{l}=0$ and $\tilde{c}_{l}=b_{l}=i^{l} / 2 \sqrt{2} \ldots$. The last part should read $\tilde{c}_{l}=b_{l}=-i^{l} / 2 \sqrt{2}$.

No other equations or figures were affected by this mistake. 